\title{
斑块面积对城市绿地降温效应的影响研究进展
}

\author{
焦 敏 ${ }^{1}$, 周伟奇 ${ }^{1,2,3, *}$, 钱雨果 ${ }^{1}$, 王 佳 ${ }^{1}$, 郑 重 $^{1}$, 胡潚方 ${ }^{1,2}$, 王伟民 ${ }^{4}$ \\ 1 中国科学院生态环境研究中心, 城市与区域生态国家重点实验室, 北京 100085 \\ 2 中国科学院大学, 北京 100049 \\ 3 中国科学院生态环境研究中心, 北京城市生态系统研究站, 北京 100085 \\ 4 深圳市环境监测中心站, 国家环境保护快速城市化地区生态环境科学观测研究站, 深圳 518049
}

摘要: 在气候变化和快速城市化的双重影响下,全球城市面临日益加剧的热岛效应。绿地能够有效改善城市热环境。城市土地 资源紧缺,优化绿地布局,使有限面积的绿地发挥更大的降温效果具有重要的理论意义和实践价值。其中,如何设置绿地斑块 大小则是优化绿地布局的首要考虑因素之一。从斑块和景观的尺度总结了现有绿地斑块大小对其降温效应影响的研究进展, 结果表明:1) 在斑块尺度, 随着绿地斑块面积增加, 绿地的降温效应相应增加, 但增加的趋势为非线性变化, 表现为温度随绿地 斑块面积的增加先下降, 后趋于平稳,表明可能存在降温效率 (单位面积绿地降温能力) 最强的斑块大小;2) 在景观尺度, 绿地 斑块大小在空间上的配置对热环境的影响研究结果存在差异,尚无一致性定论。基于已有研究,本文指出了现有绿地斑块大小 对其降温效应影响研究中的问题与不足:1) 主要关注绿地斑块大小与温度的统计关系, 缺乏对其具体影响过程和内在机制的 深人探讨;2) 更多关注地表温度, 而对与人体舒适度和健康直接相关的其他热环境指标的研究较少;3) 研究中缺少对城市中数 量巨大, 分布最广的小型绿地斑块的考量。未来的研究应加强从遮阴和蒸腾等降温过程深人解析绿地斑块大小对热环境影响 的内在机理机制, 从降温效率的角度, 探讨是否存在最优的斑块大小, 并进一步探讨绿地周围建筑环境对绿地斑块大小与其降 温效应关系的影响,为优化城市绿地分布,改善城市热环境提供科学依据。

关键词: 城市热岛; 城市绿地; 斑块大小;降温效应

\section{Influences of patch size on the cooling capacity of urban greenspace : progresses, problems and perspectives}

\footnotetext{
JIAO Min ${ }^{1}$,ZHOU Weiqi ${ }^{1,2,3, *}$, QIAN Yuguo ${ }^{1}$, WANG Jia ${ }^{1}$,ZHENG Zhong ${ }^{1}$, HU Xiaofang ${ }^{1,2}$, WANG Weimin ${ }^{4}$

1 State Key Laboratory of Urban and Regional Ecology, Research Center for Eco-Environmental Sciences, Chinese Academy of Sciences, Beijing 100085, China

2 University of Chinese Academy of Sciences, Beijing 100049, China

3 Beijing Urban Ecosystem Research Station, Research Center for Eco-Environmental Sciences, Chinese Academy of Sciences, Beijing 100085, China

4 Shenzhen Environmental Monitoring Center, State Environmental Protection Scientific Observation and Research Station for Ecology and Environment of Rapid Urbanization Region, Shenzhen 518049, China
}

\begin{abstract}
In the context of global climate change and rapid urbanization, the urban heat island effect has been aggravated in many cities around the world. Green space can effectively improve the urban thermal environment. As land resources in the city are scarce, optimizing the layout of green space and making the limited green space offer a greater cooling effect has the important theoretic significance and practical worth. How to set the green patch size is one of the first considerations for optimizing the green space pattern. In this paper, we summarized exiting research about influences of green patch area on its
\end{abstract}

基金项目: 国家自然科学基金面上项目 (41771203)

收稿日期:2020-06-19; 网络出版日期:2021-07-24

*通讯作者 Corresponding author.E-mail: wzhou@ rcees.ac.cn 
cooling effect from the patch and landscape scale, respectively. At the patch scale, the cooling effect of green space nonlinearly enhanced with increasing patch area. Specifically, as the green patch size increased, the temperature decreased firstly and then leveled off. The optimal patch size, which had the strongest cooling effect per unit area green space, may exist. At the landscape scale, effects of the spatial layout of the size of green patch on the urban thermal environment varied considerably across studies. In addition, this paper pointed out some problems and shortcomings of existing relevant studies: 1) Most of the studies focused on the statistical relationships between green patch area and temperature, while studies about the specific influence processes and mechanisms under those relationships were scare; 2) Most of the studies focused on the land surface temperature, but other thermal environmental indicators that directly related to human thermal comfort and health risk were ignored; 3 ) Small green patches, the main component of urban green space, were not included in most current studies. We further highlighted a few research directions or questions that should be paid attention in the future: 1) Analyzing the internal mechanism of effects of green patch area on urban thermal environment from the perspective of cooling processes (shade and transpiration);2) Exploring how the cooling efficiency ( cooling effect per unit area green space) change with the patch area, and detecting the optimal patch size; 3 ) Investigating influences of urban structure (building) around the green space on the relationship between green patch size and its cooling effect. To explore and/or answer these research questions can effectively further reveal specific effects of green patch size on the urban thermal environment, and thus provide important scientific bases for planning the spatial layout of urban green space, enhancing the cooling efficiency of green patches, and improving the thermal comfort of cities.

Key Words : urban heat island effect; urban green space; patch area; cooling effect

城市热岛效应是指城市温度高于周边区域的现象 ${ }^{[1]}$ 。在全球气候变化和快速城市化的双重影响下,城 市热岛效应日益加剧 ${ }^{[2]}$, 严重影响城市居民健康、热舒适度及其他城市生态功能, 是制约城市健康发展的重 要因素之一 ${ }^{[3-5]}$ 。大量研究表明, 增加城市绿地能够有效缓解热岛效应 ${ }^{[6-7]}$ 。同时, 绿地的空间配置, 如斑块 大小、形状、空间邻接关系等对城市热环境也有显著影响 ${ }^{8-10]}$ 。城市内土地资源有限, 绿地建设成本高, 相比 于一味单纯地增加绿地占地面积, 优化绿地景观格局, 提升单位面积绿地的降温能力, 使有限面积的绿地发挥 最大的降温效果具有更加重要的理论意义和实践价值,也是目前城市热环境研究关注的热点。

在众多的绿地空间配置特征中, 斑块大小是绿地规划与景观设计中考虑的首要因子之一, 其对城市热环 境的影响, 已在国内外诸多城市开展了大量的研究。结果一致表明绿地斑块面积越大, 绿地本身温度越低, 为 周围环境提供的降温效果更强 (包括降温强度和影响范围) ${ }^{[8,11-14]}$ 。但随着斑块面积的增加, 绿地整体降温 效应呈现何种增长模式? 单位面积绿地提供的降温能力 (即降温效率) 是否也相应持续增加? 总面积一定的 情况下, 规划成一个面积较大的绿地斑块, 或是几个面积较小的绿地斑块, 哪种配置方式具有更好的降温效 果? 这些研究问题目前尚未有明确的结论。本文将在系统梳理国内外相关研究的基础上,探讨绿地斑块大小 对其降温效应的影响,以期为优化城市绿地斑块大小配置,改善城市热环境提供科学指导与理论支撑。

\section{1 国内外研究现状}

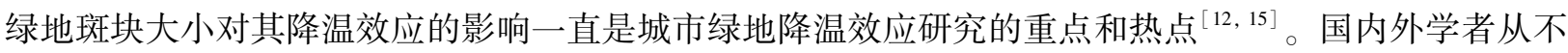
同的角度、在不同的城市, 开展了大量的相关研究。概括起来, 这些研究分别从斑块和景观两个尺度上开展: (1) 在斑块尺度上, 主要探究单个绿地斑块的大小对其降温效应的影响; (2) 在景观尺度上, 主要关注绿地斑 块大小在空间上的配置(通常采用平均斑块面积等景观指数表征)对城市热环境的影响。

\section{1 斑块尺度: 绿地斑块面积对其降温效应的影响}

在斑块尺度, 现有研究关注的绿地斑块分别为: 包含其他地表覆盖在内的复合型绿地斑块 (如公园) 和仅 由绿地组成的单一绿地斑块, 研究这两种类型绿地玟块面积对绿地本身及其周围环境温度的影响, 大部分的 
研究结果均显示随着斑块面积的增加, 绿地的降温效应相应增强。

1.1.1 复合型绿地斑块大小与其降温效应正相关, 但同时受内部其他地表覆盖组成的影响

早期绿地斑块大小与其降温效应的大量研究主要关注公园类的复合型绿地。研究结果表明面积对公园 绿地的降温效应具有重要的影响, 表现为大面积的公园绿地自身温度更低, 且对周围环境具有更强的降温效 应 $^{[15-16]}$ 。例如,塞俄比亚的亚的斯亚贝巴 21 个城市公园气温及地表温度的测量结果显示, 面积越大的公园 绿地降温效应越明显, 其对周围环境的降温幅度更大、降温距离更远 ${ }^{[17]}$ 。类似的, 比较台北 61 个公园绿地夏 季正午的温度, 发现面积超过 $30000 \mathrm{~m}^{2}$ 的公园绿地具有更强的降温效应 ${ }^{[8]}$ 。在北京和上海开展的公园绿地 降温效应的研究中也发现公园绿地面积增加, 其降温效应也相应增加 ${ }^{[12,16,18]}$ 。需要指出的是, 公园类复合型 绿地由于内部结构复杂且包含多种地表类型, 其大小与降温效应的关系, 会受到公园内部各种类型地表的覆 盖比例, 以及植被组成结构等因素的影响 ${ }^{[19-21]}$ 。例如, 随着公园内植被覆盖比例的增加, 公园的降温效应会 加强。有研究发现即使面积不变, 但当香港的 “口袋公园” (面积约为 $1000 \mathrm{~m}^{2}$ 的小型公园) 内植被覆盖比例从 $25 \%$ 增加至 $40 \%$ 时, 其降温幅度将增加 $0.5^{\circ} \mathrm{C}^{[22]}$ 。更有研究发现当公园内不透水地表的比例超过 $50 \%$ 时, 公 园不再具有降温作用,从“冷岛” 变为了 “热岛” ${ }^{[8]}$ 。而将公园内的草地替换为林地,也能有效提升该公园的降 温效应 ${ }^{[23]}$ 。

1.1.2 绿地的降温效应随着面积变大呈非线性增强, 可能存在最佳斑块面积

以单一的绿地为研究对象展开的绿地斑块大小与其降温效应关系的研究也得到了与上述公园类复合型 绿地相似的结果, 即绿地斑块面积越大, 其降温效应越强 ${ }^{[24-25]}$ 。随着绿地斑块面积的增加, 其内部核心低温 区域所占的面积比例增加 ${ }^{[26-27]}$, 因此绿地斑块的整体温度相应降低, 并对周围环境产生更强的降温效 果 ${ }^{[25,28-33]}$ 。但是, 绿地斑块大小与其降温效应 (包括降温幅度和降温影响空间范围) 并非线性关系 ${ }^{[34-35]}$ 。这 一现象无论是在公园类复合型绿地,还是单一绿地斑块的研究中均有发现。对长春市的 33 个公园绿地的研 究发现, 在绿地面积较小时随着面积的增大, 其降温强度逐渐增强, 但当绿地面积增大到一定阈值之后, 其降 温强度不再有明显的上升趋势 ${ }^{[15]}$ 。不仅仅是降温强度, 对广州 10 个公园绿地的研究发现绿地对周围环境的 降温影响空间范围也表现出相同的现象, 随着绿地面积的增加, 其降温距离也相应增加, 但降温距离增加的速 度 (单位面积绿地增加导致的降温距离增加) 逐渐变缓, 当绿地面积增大到 $740000 \mathrm{~m}^{2}$ 之后, 面积每再增加 $10000 \mathrm{~m}^{2}$, 降温距离往外延伸不足 $1 \mathrm{~m}^{[36]}$ 。对韩国蔚山市内 30 个不同类型的绿地斑块展开的研究中也发现, 绿地斑块面积与其降温效应呈非线性的正相关 ${ }^{[37]}$ 。对福州整个城市内的绿地斑块开展的研究发现当绿地斑 块面积大于 $45500 \mathrm{~m}^{2}$ 这一阈值之后, 随着绿地斑块面积的增加, 其降温幅度已不再具有明显的增加趋势 ${ }^{[38]}$ 。 此外,该阈值在不同的气候背景或城市结构中是不同的,亚洲 7 个不同城市的对比研究发现在背景气候温度 越高、绿地越丰富 (NDVI 值高) 的城市内 (高雄、吉隆坡和台南) 阈值越大 (9200- $9600 \mathrm{~m}^{2}$ ), 而在背景气候温 度较低且绿地相对贫痊的城市内 (香港、雅加达、孟买和新加坡) 阈值相对较小 $\left(6000-6200 \mathrm{~m}^{2}\right)^{[39]}$ 。这些研 究结果均表明, 从降温效率 (单位面积绿地提供的降温效应) 的角度而言, 可能存在最佳绿地斑块面积。一项 在北京开展的单位面积绿地提供的遮阴和蒸腾降温效应随绿地斑块面积变化的研究发现, 随着绿地斑块面积 增加, 单位面积绿地提供的遮阴持续降低, 但单位面积绿地提供的蒸腾则随着斑块面积呈现非线性的变化, 也 证实在大绿地斑块和小绿地斑块之间存在着降温效率最高的最佳绿地斑块面积 ${ }^{[40]}$ 。

1.2 景观尺度:绿地斑块大小空间配置对城市热环境的影响

在景观尺度上, 现有研究主要关注绿地斑块大小在空间上的分布格局对绿地本身以及整体景观环境温度 的影响。而绿地斑块大小在空间上的分布格局主要通过各种景观格局指数来刻画。此外,也有部分研究直接 在模型中设置不同的格局情景。不同于斑块尺度上研究结果的一致性, 景观尺度上绿地斑块大小空间分布情 况对热环境的影响在不同的研究中呈现不同、甚至相反的结果。

1.2.1 绿地斑块大小空间分布对城市热环境的影响在不同的研究中存在明显差异

基于绿地景观格局指数和温度的统计分析是目前景观尺度上绿地斑块面积对热环境影响的主要研究方 
法。通过景观格局指数来表征绿地斑块大小在空间上的分布情况,进而分析这些景观格局指数与温度之间的 统计关系 ${ }^{[41-44]}$, 其中常见用于表征绿地斑块大小空间分布情况的景观指数如下表 1 所示。已有的大部分研 究均发现绿地平均斑块面积 (AREA_MN) 和最大斑块面积指数 (LPI) 与绿地本身或其周围环境的温度呈显著 负相关, 即在某一区域内绿地斑块越大,绿地本身及该区域的整体环境温度越低 ${ }^{[45-55]}$ 。但也有部分研究发现 了相反的结果, 这两个景观格局指数与温度呈显著正相关 ${ }^{[12,48,56]}$ 。关于少量的大绿地斑块和多个破碎的小 绿地斑块, 哪种格局具有更强的降温效应, 不同的研究也分别发现了不同的结果。部分研究显示, 大面积绿地 斑块变为多个小绿地斑块会导致城市地表温度的显著上升,即大绿地斑块比多个破碎的小绿地斑块对整体热 环境具有更好的降温效果 ${ }^{[30,41,57-58]}$, 如对成都内绿地覆盖率相同的两个区域进行比较, 发现虽然绿地覆盖率 相同,但绿地斑块面积较大且分布相对集中的区域的温度明显低于绿地斑块面积较小且分布相对分散的区 域 ${ }^{[59]}$ 。对上海林地开展的一项研究也发现, 在林地覆盖度相同的情况下,绿地聚集分布比碎片化分布具有更 好的降温效应 ${ }^{[60]}$ 。这是由于大绿地斑块比小绿地斑块具有更强的降温效果 ${ }^{[43]}$ 。但也有研究显示在绿地总 面积固定时, 分散的多个小绿地斑块比少量且聚集的大斑块能更有效地缓解城市热环境 ${ }^{[42]}$, 分散的多个小绿 地斑块虽然每个小斑块本身的“冷岛效应”较弱,但是这种格局因为具有更多的边缘,可以给周围环境提供更 多的遮阴,并且促进绿地和周围环境之间的热量流通, 从而使整体环境温度更低 ${ }^{[61]}$ 。

表 1 表征绿地斑块大小空间分布的常用景观指数

Table 1 Landscape metrics that commonly used for charactering the spatial pattern of green patch size

\begin{tabular}{|c|c|c|c|}
\hline $\begin{array}{l}\text { 景观指数 } \\
\text { Landscape metrics }\end{array}$ & $\begin{array}{l}\text { 缩写 } \\
\text { Abbreviation }\end{array}$ & $\begin{array}{l}\text { 描述 } \\
\text { Description }\end{array}$ & $\begin{array}{l}\text { 参考文献 } \\
\text { References }\end{array}$ \\
\hline $\begin{array}{l}\text { 平均斑块面积 } \\
\text { Mean patch area }\end{array}$ & AREA_MN & $\begin{array}{l}\text { 绿地斑块的平均面积, 等于绿地斑块总面积除以 } \\
\text { 斑块总数量 }\end{array}$ & {$[45-47]$} \\
\hline $\begin{array}{l}\text { 面积加平均斑块面积 } \\
\text { Area-weighted mean patch area }\end{array}$ & AREA_AM & $\begin{array}{l}\text { 考虑了各个斑块面积所占权重的绿地斑块平均 } \\
\text { 面积 }\end{array}$ & {$[41]$} \\
\hline $\begin{array}{l}\text { 最大斑块面积指数 } \\
\text { Largest patch index }\end{array}$ & LPI & $\begin{array}{l}\text { 最大斑块占景观总面积的比例, 等于最大斑块的 } \\
\text { 面积除以景观总面积 }\end{array}$ & {$[46,48]$} \\
\hline $\begin{array}{l}\text { 斑块大小标准差 } \\
\text { Patch size standard deviation }\end{array}$ & AREA_SD & $\begin{array}{l}\text { 景观内斑块大小分布的标准差, 表征斑块大小的 } \\
\text { 绝对离散程度 }\end{array}$ & {$[9,48]$} \\
\hline $\begin{array}{l}\text { 斑块大小变异系数 } \\
\text { Patch size coefficient of variation }\end{array}$ & AREA_CV & $\begin{array}{l}\text { 景观内斑块大小的变异系数, 表征斑块大小的相 } \\
\text { 对离散程度,消除了平均斑块大小的影响 }\end{array}$ & {$[48]$} \\
\hline $\begin{array}{l}\text { 核心区比例 } \\
\text { Core area percent of landscape }\end{array}$ & CPLAND & $\begin{array}{l}\text { 所有绿地斑块核心区总面积占景观总面积的比 } \\
\text { 例, 表征景观中绿地斑块大小分布情况 }\end{array}$ & {$[49]$} \\
\hline
\end{tabular}

此外,也有基于模型模拟进行的景观尺度上绿地玟块面积对城市热环境影响的研究,如模拟局地微气候 的 ENVI-met 模型、局地尺度城市气象参数模型 (Local-Scale Urban Meteorological Parameterization Scheme, LUMPS)、城市森林效益评估 i-Tree 模型等 ${ }^{[62-64]}$ 。这类研究主要通过人为在模型中设置不同的情景模式, 对 比研究不同的绿地斑块大小分布情况对热环境的影响差异, 能够有效地控制其他影响热环境的变量, 针对性 分析绿地斑块大小对热环境的影响, 并且可以脱离样本获取难度对研究的限制, 设置多种不同的绿地斑块大 小分布情景进行研究。但由于模型模拟具有较多的物理假设及约束条件, 且城市景观格局和局地微气候极为 复杂,模型模拟难以全面考虑现实环境中局地景观和气候特征的异质性,模拟结果与实际情况有较大差异,因 而模型模拟的应用也存在诸多限制。一项在台北开展的相关研究, 选取 $1710000 \mathrm{~m}^{2}$ 的典型城市化区域为研究 对象,采用 ENVI-met 分别构建 8 种不同的绿地斑块大小组成情景, 并对比研究其彼此间的降温效应差异,结 果显示在绿地总面积固定的前提下,构建一个大型绿地并置于下风区或者构建多个小绿地并使其尽量均匀地 分布于整个区域,均能使固定面积的绿地发挥更强的降温效果 ${ }^{[65]}$ 。另一项同样在台北开展,采用 LES-LSM (large-eddy simulation land surface model) 模型进行的类似研究, 则发现随着单个大绿地斑块逐渐分散成多个 小斑块,整体区域的地表温度和气温都逐渐升高 ${ }^{[66]}$ 。

1.2.2 绿地斑块大小空间分布对城市热环境的影响在不同的研究中存在差异的主要原因

如上所述, 绿地斑块大小空间配置对城市热环境的影响在不同的研究中出现不同、甚至完全相反的结果。 
这可能主要是由于如下 4 个方面的原因导致的: (1) 不同研究中量化绿地斑块大小空间配置所采用的遥感数 据空间分辨率不同。一方面,空间分辨率的不同会导致格局指数本身的数值发生较大的变化,例如空间分辨 率越粗粘时,平均斑块面积越大。这是由于在数据空间分辨率较粗粘时,多个相距较近的小绿地被识别和提 取为一个大绿地斑块 ${ }^{[47]}$ 。另一方面,空间分辨率的差异也影响着参与研究分析的绿地大小等级,在空间分辨 率较粗鉌时, 由于很多小绿地斑块不能被识别和提取, 故而未能参与最终分析。反之, 精细的遥感数据能够准 确识别和提取小绿地斑块, 从而大大增加了研究样本中小绿地斑块的比例 ${ }^{[47]} ;$; 2) 不同研究中采取的分析方 法不同。一项在北京开展的研究发现,控制绿地比例与否会导致绿地格局对热环境的影响发生变化, 例如在 控制绿地比例之后, 绿地平均斑块面积与地表温度的相关性变弱 ${ }^{[47]}$ 。而另一项在美国巴尔的摩展开的研究 则发现, 在控制林地比例之后, 绿地平均斑块面积与地表温度的相关性由原本的负相关变为正相关 ${ }^{[4]}$; (3) 研 究区域气候背景以及城市结构的差异。目前不同城市之间的对比研究逐渐增多, 这些城市位于不同气候区 域,或规模大小、内部结构有明显差异,即使采用相同的数据和研究方法, 不同城市之间的研究结果也具有明 显, 甚至是完全相反的差异 ${ }^{[67-70]}$ 。例如对新加坡、香港、雅加达和吉隆坡开展的对比研究发现, 除香港外, 其 他三个城市呈现出背景气候温度越高, 绿地平均斑块大小与温度之间的负相关性越强的现象 ${ }^{[67]}$ 。一项美国 巴尔的摩和萨克拉门托两个城市的对比研究也发现, 城市林地平均斑块大小与地表温度在巴尔的摩呈现显著 的正相关, 但在萨克拉门托则呈现显著的负相关, 其结果的差异, 可能与两个城市的林地破碎化程度, 以及不 同的气候背景有关 ${ }^{[44]}$ 。另一项对同一地区 (北京) 不同季节开展的对比研究也验证了气候背景对 “绿地斑块 大小与其降温效应关系” 存在影响, 该研究结果显示绿地斑块大小与其本身地表温度的负相关性在温度较高 的季节更强 ${ }^{[25]}$, 这可能是由于绿地在背景气候温度更高的时候表现出更强的降温效应 ${ }^{[71]}$; (4) 这种不一致 性, 也可能与研究对象, 即地表温度和气温本身的特征有关。温度具有高空间异质性、高动态度的特点, 且易 受人为和气象因素影响 ${ }^{[72-75]}$ 。地表温度的研究通常是利用遥感反演出某个时间点的温度特征, 无法反映温 度在时间上的累积动态。而气温往往是基于定点监测或流动监测, 虽能反映点和线的温度特征, 却难以确定 温度所能代表的空间范围, 难以厘定绿地对气温的贡献程度 ${ }^{[73]}$ 。

\section{2 现有研究存在的问题与不足}

城市绿地斑块大小对其降温效应的影响得到了广泛的关注, 并开展了大量的相关研究。但是, 现有的研 究主要集中关注绿地斑块大小与温度的统计关系, 缺乏对其降温过程和内在机制的探讨, 并且多数研究主要 是针对地表温度, 而对与人体热舒适度和健康直接相关的其他热环境指标则关注较少。此外, 由于现有研究 数据空间分辨率的限制, 城市内大量的小型绿地斑块未能参与研究, 导致现有对绿地斑块大小与热环境关系 的认识并不全面。具体而言,主要包括以下 3 个方面的问题和挑战。

2.1 主要关注绿地斑块大小与温度的统计关系, 缺乏对其降温过程和内在机制的探讨

大量的城市绿地斑块大小与其降温效应关系的研究, 主要关注两者的统计关系, 缺乏对其降温过程和内 在机制的探讨,未能有效揭示城市绿地斑块大小对温度影响的具体原因。例如,在景观尺度上的分析,已有的 研究得出了不一致、甚至是完全相反的结果。那么, 这种情况的出现, 是因为所采用的数据和分析方法的不 同,还是绿地的降温机制在不同的城市确实存在差异? 这些问题,需要通过绿地降温过程和机制的研究来回 答。目前开展的绿地降温机理的研究, 主要集中在个体 (单株) 尺度, 探讨通过冠层提供的遮阴来减少太阳辐 射的输人, 以及植株的蒸腾作用来增加太阳辐射中潜热的转化比例来实现降温作用的过程和机理 ${ }^{[76-80]}$, 而在 斑块和景观尺度上的探讨绿地斑块大小影响其降温效应的具体过程和内在机制很少,急需加强。

2.2 更关注地表温度, 而对与人体热舒适度和健康直接相关的其他热环境指标的研究较少

当前, 全球面临着快速的城市化过程, 已有超过 $50 \%$ 的人口居住在城市中 ${ }^{[81]}$ 。城市中的热环境对人类居 住舒适度以及身体健康有重要影响 ${ }^{[82-83]}$, 极端热环境条件 (如热浪) 会导致人类心血管等疾病的发生率甚至 死亡率的增加 ${ }^{[7]}$ 。现有绿地斑块大小对热环境影响的相关研究大多基于地表温度来展开, 探讨绿地斑块大 
小对绿地本身及景观环境的表面温度的影响。但对于更能直接表征人类居住舒适度以及健康风险的气温、热 舒适度、辐射温度、生理等效温度 ${ }^{[84-85]}$ 等的研究则较少, 这使得现有研究结果在应用于指导优化绿地格局, 从 而提升城市热舒适度,降低热浪对人类的健康威胁上仍有一定的距离。

\section{3 研究中缺少城市小绿地斑块的参与}

目前城市绿地的提取和量化主要采用的是中等空间分辨率 $(30 \mathrm{~m})$ 的 Landsat 数据 $(\mathrm{TM}, \mathrm{ETM}+$ 和 landsat 8 ), 但城市内的绿地高度破碎化, 小型绿地斑块是城市绿地的主体, 如北京有 $76.7 \%$ 的绿地斑块小于 $900 \mathrm{~m}^{2}$ (2009 年数据) ${ }^{[86]}$, 这些绿地斑块由于小于单个像元, 因此无法被识别和提取。此外, 从遥感影像地物识别和 提取技术上而言, 斑块面积需大于遥感像元 4 倍 $\left(3600 \mathrm{~m}^{2}\right)$ 以上时, 地物斑块才能易于被识别和提取出来 ${ }^{[87]}$, 这使得城市内更多的绿地无法被准确识别和提取,如北京内面积小于 $3600 \mathrm{~m}^{2}$ 的绿地斑块所占据的比例高达 $91.9 \%{ }^{[86]}$ 。虽然目前绿地的提取和量化采用高空间分辨率数据的频率有所增加, 如 SPOT 和 Quickbird ${ }^{[47]}$, 但 中等空间分辨率的数据依然占据主体。除绿地提取和量化之外,绿地表面温度的刻画更是主要采用中等分辨 率的 landsat TM, ETM+, landsat 8 和 ASTER 的热红外波段(空间分辨分别为 $120,60,100 \mathrm{~m}$ 和 $90 \mathrm{~m})^{[12,27]}$, 仅有极少数的研究通过航拍的方法获取较高空间分辨率的地表温度数据 ${ }^{[88-89]}$, 这使得即使绿地的提取精度 有所提升, 小绿地斑块能够被更加准确地识别和提取出来, 但由于地表温度数据的分辨率限制, 小绿地斑块依 然未能参与最终的研究分析。从而导致现有研究中缺失了小型绿地这一城市绿地类型的主体, 对绿地斑块大 小与城市热环境关系的研究结果将有重要影响。

\section{3 研究展望}

快速的城市化依然是我国未来较长一段时间内城市发展的主要趋势, 这也将进一步加剧城市热岛效应及 其相应导致的一系列城市生态环境问题,降低城市人居舒适度,影响居民身体健康,这些都是我国城市可持续 发展目前面临的重大挑战。因此深人研究绿地对城市热环境的具体影响,探寻绿地最优分布格局,提升绿地 的降温效率, 使有限面积的绿地发挥最大的降温效果, 从而切实有效地改善城市热环境是应对这些挑战的重 要措施。具体而言,绿地斑块大小对城市热环境影响的研究仍需在以下几个方面进行进一步的深人分析。

(1) 斑块面积对绿地降温效率的定量影响。虽然增加绿地面积能够有效改善城市热环境,但是城市由于 受到多重社会及经济条件的制约,绿地建设具有很高的成本, 相比于一味单纯地增加绿地面积,在增加城市绿 地面积的同时, 提升单位面积绿地的降温能力, 使一定面积的绿地能发挥最大的降温效果则能够更加有效地 改善城市热环境,维持城市的可持续发展。因此,定量分析绿地斑块面积对单位面积绿地降温能力 (降温效 率) 的影响, 探寻具有最大降温效率的绿地斑块大小 (最佳绿地斑块大小) 是绿地斑块大小对城市热环境影响 的研究中急需要进一步深人的重要方向。

(2) 绿地斑块面积对降温过程以及相应导致的能量变化的影响。分析绿地斑块面积与温度的统计关系 仅能够分析绿地斑块面积对其降温效应影响的表观结果, 不足以揭示其内在机制, 对于进一步深人理解绿地 斑块面积对其降温效应的具体影响,进而应用于绿地规划产生了较大的限制。城市热环境主要是由地表能量 输人与输出决定, 因此从地表能量平衡的角度, 综合考虑绿地的两个关键降温过程——蒸腾和遮阴 ${ }^{[90]}$, 分析 随着绿地斑块面积变化, 蒸腾和遮阴降温过程相应导致的能量变化, 能够从机制上对绿地斑块面积与降温效 应关系进行研究,并且也有利于不同气候背景下研究结果的互相比较。

(3) 周围建筑环境对绿地斑块大小与其降温效应关系的影响。绿地的降温效应受到温度、湿度、风速和 太阳辐射等微气象背景环境因子的影响 ${ }^{[91]}$, 而这些因子又受到一定区域内建筑空间环境的影响 ${ }^{[92]}$, 因此, 绿 地斑块大小对其降温效果的研究,应综合考虑绿地所在的一定空间区域范围内城市空间形态的影响。耦合绿 地形态与建筑空间的热效应研究具有重要的理论与现实意义。绿地降温效应受建筑群空间形态的影响, 主要 包括两个方面, 其中直接的影响, 主要是建筑对绿地提供的遮阴降温过程的影响。建筑由于能够提供比绿地 强度更大的遮阴, 因而绿地周围的建筑会在一定的程度上影响绿地遮阴降温效果 ${ }^{[93]}$ 。而间接的影响, 主要是 
建筑和绿地共同作用影响局地微气候,如气温、湿度和风场等,从而影响绿地蒸腾降温作用。

\section{参考文献( References) :}

[ 1 ] Voogt J A, Oke T R. Thermal remote sensing of urban climates. Remote Sensing of Environment, 2003, 86(3) : 370-384.

[ 2 ] Zhou W Q, Qian Y G, Li X M, Li W F, Han L J. Relationships between land cover and the surface urban heat island: seasonal variability and effects of spatial and thematic resolution of land cover data on predicting land surface temperatures. Landscape Ecology, 2014, 29(1) : $153-167$.

[ 3 ] Santamouris M, Kolokotsa D. Passive cooling dissipation techniques for buildings and other structures: the state of the art. Energy and Buildings, 2013, 57: 74-94.

[ 4 ] Sun S Z, Cao P H, Chan K P, Tsang H, Wong C M, Thach T Q. Temperature as a modifier of the effects of fine particulate matter on acute mortality in Hong Kong. Environmental Pollution, 2015, 205: 357-364.

[ 5 ] Phung D, Guo Y M, Tai P, Rutherford S, Wang X M, Nguyen M, Do C M, Nguyen N H, Alam N, Chu C. The effects of high temperature on cardiovascular admissions in the most populous tropical city in Vietnam. Environmental Pollution, 2016, 208: 33-39.

[ 6 ] Chiesura A. The role of urban parks for the sustainable city. Landscape and Urban Planning, 2004, 68(1) : 129-138.

[ 7 ] Bowler D E, Buyung-Ali L, Knight T M, Pullin A S. Urban greening to cool towns and cities: a systematic review of the empirical evidence. Landscape and Urban Planning, 2010, 97(3) : 147-155.

[ 8 ] Chang C R, Li M H, Chang S D. A preliminary study on the local cool-island intensity of Taipei city parks. Landscape and Urban Planning, 2007, $80(4)$ : 386-395.

[ 9 ] Zhou W Q, Huang G L, Cadenasso M L. Does spatial configuration matter? Understanding the effects of land cover pattern on land surface temperature in urban landscapes. Landscape and Urban Planning, 2011, 102(1) : 54-63.

[10］袁振, 吴相利, 藏淑英, 吴长山, 李苗. 基于 TM 影像的哈尔滨市主城区绿地降温作用研究. 地理科学, 2017, 37(10): 1600-1608.

[11] Adams M P, Smith P L. A systematic approach to model the influence of the type and density of vegetation cover on urban heat using remote sensing. Landscape and Urban Planning, 2014, 132: 47-54.

[12] Cheng X Y, Wei B S, Chen G J, Li J X, Song C H. Influence of park size and its surrounding urban landscape patterns on the park cooling effect. Journal of Urban Planning and Development, 2015, 141(3): A4014002.

[13] Guo G H, Wu Z F, Xiao R B, Chen Y B, Liu X N, Zhang X S. Impacts of urban biophysical composition on land surface temperature in urban heat island clusters. Landscape and Urban Planning, 2015, 135: 1-10.

[14] Sun X, Tan X Y, Chen K L, Song S, Zhu X D, Hou D L. Quantifying landscape-metrics impacts on urban green-spaces and water-bodies cooling effect: the study of Nanjing, China. Urban Forestry \& Urban Greening, 2020, 55: 126838.

[15] Ren Z B, He X Y, Zheng H F, Zhang D, Yu X Y, Shen G Q, Guo R C. Estimation of the relationship between urban park characteristics and park cool island intensity by remote sensing data and field measurement. Forests, 2013, 4(4): 868-886.

[16］孟丹, 李小娟, 宫辉力, 赵文吉. 北京地区热力景观格局及典型城市景观的热环境效应. 生态学报, 2010, 30(13): 3491-3500.

[17] Feyisa G L, Dons K, Meilby H. Efficiency of parks in mitigating urban heat island effect: an example from Addis Ababa. Landscape and Urban Planning, 2014, 123: 87-95.

[18] 徐丽华, 岳文泽. 城市公园景观的热环境效应. 生态学报, 2008, 28(4)：1702-1710.

[19］冯悦怡, 胡潭高, 张力小. 城市公园景观空间结构对其热环境效应的影响. 生态学报, 2014, 34(12) : 3179-3187.

[20］赵芮, 申全金, 田国行, 郭显琛, 何瑞珍. 郑州市公园绿地景观特征对公园冷岛效应的影响. 生态学报, 2020, 40(9): $2886-2894$.

[21] Li H W, Wang G F, Tian G H, Jombach S. Mapping and analyzing the park cooling effect on urban heat island in an expanding city : a case study in Zhengzhou city, China. Land, 2020, 9(2): 57.

[22] Giridharan R, Lau S S Y, Ganesan S, Givoni B. Lowering the outdoor temperature in high-rise high-density residential developments of coastal Hong Kong: the vegetation influence. Building and Environment, 2008, 43(10): 1583-1595.

[23] Potchter O, Cohen P, Bitan A. Climatic behavior of various urban parks during hot and humid summer in the Mediterranean city of Tel Aviv, Israel. International Journal of Climatology, 2006, 26(12): 1695-1711.

[24] Hirano Y, Yasuoka Y, Ichinose T. Urban climate simulation by incorporating satellite-derived vegetation cover distribution into a mesoscale meteorological model. Theoretical and Applied Climatology, 2004, 79(3/4): 175-184.

[25] Chen A L, Yao X A, Sun R H, Chen L D. Effect of urban green patterns on surface urban cool islands and its seasonal variations. Urban Forestry \& Urban Greening, 2014, 13(4) : 646-654.

[26] Zhang X Y, Zhong T Y, Feng X Z, Wang K. Estimation of the relationship between vegetation patches and urban land surface temperature with remote sensing. International Journal of Remote Sensing, 2009, 30(8) : 2105-2118. 
[27] Gioia A, Paolini L, Malizia A, Oltra-Carrió R, Sobrino J A. Size matters: vegetation patch size and surface temperature relationship in foothills cities of northwestern Argentina. Urban Ecosystems, 2014, 17(4) : 1161-1174.

[28] Srivanit M, Iamtrakul P. Spatial patterns of greenspace cool islands and their relationship to cooling effectiveness in the tropical city of Chiang Mai, Thailand. Environmental Monitoring and Assessment, 2019, 191(9) : 580.

[29] 陈爱莲, 孙然好, 陈利顶. 绿地格局对城市地表热环境的调节功能. 生态学报, 2013, 33(8): 2372-2380.

[30] Masoudi M, Tan P Y. Multi-year comparison of the effects of spatial pattern of urban green spaces on urban land surface temperature. Landscape and Urban Planning, 2019, 184: 44-58.

[31] Ersoy E. Landscape pattern and urban cooling islands. Fresenius Environmental Bulletin, 2019, 28 (3) : $1943-1951$.

[32] Yang C B, He X Y, Wang R H, Yan F Q, Yu L X, Bu K, Yang J C, Chang L P, Zhang S W. The effect of urban green spaces on the urban thermal environment and its seasonal variations. Forests, 2017, 8(5): 153.

[33] 李孝永, 匡文慧. 北京、天津和石家庄城市地表覆盖组分与结构特征对地表温度的影响. 生态学杂志, 2019, 38(10): 3057-3065.

[34] Monteiro M V, Doick K J, Handley P, Peace A. The impact of greenspace size on the extent of local nocturnal air temperature cooling in London. Urban Forestry \& Urban Greening, 2006, 16: 160-169.

[35] Lin W Q, Yu T, Chang X Q, Wu W J, Zhang Y. Calculating cooling extents of green parks using remote sensing: method and test. Landscape and Urban Planning, 2015, 134: 66-75.

[36] Chen X Z, Su Y X, Li D, Huang G Q, Chen W Q, Chen S S. Study on the cooling effects of urban parks on surrounding environments using Landsat TM data: a case study in Guangzhou, southern China. International Journal of Remote Sensing, 2012, 33( 18) : 5889-5914.

[37] Park J H, Cho G H. Examining the Association between physical characteristics of green space and land surface temperature: a case study of Ulsan, Korea. Sustainability, 2016, 8(8): 777 .

[38] Yu Z W, Guo X Y, Jørgensen G, Vejre H. How can urban green spaces be planned for climate adaptation in subtropical cities? Ecological Indicators, 2017, 82: 152-162.

[39] Fan H Y, Yu Z W, Yang G Y, Liu T Y, Liu T Y, Hung C H, Vejre H. How to cool hot-humid (Asian) cities with urban trees? An optimal landscape size perspective. Agricultural and Forest Meteorology, 2019, 265: 338-348.

[40] Jiao M, Zhou W Q, Zheng Z, Wang J, Qian Y G. Patch size of trees affects its cooling effectiveness: a perspective from shading and transpiration processes. Agricultural and Forest Meteorology, 2017, 247: 293-299.

[41] Liu H L, Shen Y S. The impact of green space changes on air pollution and microclimates: a case study of the Taipei metropolitan area. Sustainability, 2014, 6(12): 8827-8855.

[42] Zhang Y Z, Zhan Y L, Yu T, Ren X Y. Urban green effects on land surface temperature caused by surface characteristics: a case study of summer Beijing metropolitan region. Infrared Physics \& Technology, 2017, 86: 35-43.

[43] Ren Z B, Zheng H F, He X Y, Zhang D, Yu X Y. Estimation of the relationship between urban vegetation configuration and land surface temperature with remote sensing. Journal of the Indian Society of Remote Sensing, 2015, 43(1) : 89- 100.

[44] Zhou W Q, Wang J, Cadenasso M L. Effects of the spatial configuration of trees on urban heat mitigation: a comparative study. Remote Sensing of Environment, 2017, 195: 1-12.

[45] Li X M, Zhou W Q, Ouyang Z Y, Xu W H, Zheng H. Spatial pattern of greenspace affects land surface temperature: evidence from the heavily urbanized Beijing metropolitan area, China. Landscape Ecology, 2012, 27(6) : 887-898.

[46] Kong F H, Yin H W, James P, Hutyra L R, He H S. Effects of spatial pattern of greenspace on urban cooling in a large metropolitan area of eastern China. Landscape and Urban Planning, 2014, 128: 35-47.

[47] Li X M, Zhou W Q, Ouyang Z Y. Relationship between land surface temperature and spatial pattern of greenspace: what are the effects of spatial resolution? Landscape and Urban Planning, 2013, 114: 1-8.

[48] Weber N, Haase D, Franck U. Zooming into temperature conditions in the city of Leipzig: how do urban built and green structures influence earth surface temperatures in the city? Science of the Total Environment, 2014, 496: 289-298.

[49] Sun R H, Lü Y H, Chen L D, Yang L, Chen A L. Assessing the stability of annual temperatures for different urban functional zones. Building and Environment, 2013, 65: 90-98.

[50] Ghosh S, Das A. Modelling urban cooling island impact of green space and water bodies on surface urban heat island in a continuously developing urban area. Modeling Earth Systems and Environment, 2018, 4(2) : 501-515.

[51] Amani-Beni M, Zhang B, Xie G D, Shi Y T. Impacts of urban green landscape patterns on land surface temperature: evidence from the adjacent area of Olympic forest park of Beijing, China. Sustainability, 2019, 11(2) : 513.

[52] Li B, Shi X M, Wang H Y, Qin M Z. Analysis of the relationship between urban landscape patterns and thermal environment: a case study of Zhengzhou City, China. Environmental Monitoring and Assessment, 2020, 192(8) : 540. 
[53] 雷金睿, 陈宗铸, 吴庭天, 李苑菱, 陈小花, 杨琦, 何荣晓. 1989-2015 年海口城市热环境与景观格局的时空演变及其相互关系. 中国环 境科学, 2019, 39(4): 1734-1743.

[54] Song Y, Song X D, Shao G F. Effects of green space patterns on urban thermal environment at multiple spatial-temporal scales. Sustainability, $2020,12(17): 6850$.

[55] Nastran M, Kobal M, Eler K. Urban heat islands in relation to green land use in European cities. Urban Forestry \& Urban Greening, 2019, 37: 3341.

[56] Zhang Y S, Odeh I O A, Ramadan E. Assessment of land surface temperature in relation to landscape metrics and fractional vegetation cover in an urban/peri-urban region using Landsat data. International Journal of Remote Sensing, 2013, 34(1) : 168-189.

[57] 周雯, 曹福亮, 张瑞, 汪贵斌. 绿地格局对城市地表热环境调节作用的多尺度分析. 南京林业大学学报: 自然科学版, 2020, 44(3)： 133-141.

[58］高美蓉, 贾宝全, 王成, 孙朝晖. 厦门本岛城市森林树冠覆盖与热岛效应关系. 林业科学, 2014, 50(3) : 63-68,

[59］陈辉, 古琳, 黎燕琼, 慕长龙. 成都市城市森林格局与热岛效应的关系. 生态学报, 2009, 29(9): 4865-4874.

[60] Zhou W, Cao F L, Wang G B. Effects of spatial pattern of forest vegetation on urban cooling in a compact megacity. Forests, 2019, 10(3) : 282.

[61] Maimaitiyiming M, Ghulam A, Tiyip T, Pla F, Latorre-Carmona P, Halik Ü, Sawut M, Caetano M. Effects of green space spatial pattern on land surface temperature: implications for sustainable urban planning and climate change adaptation. ISPRS Journal of Photogrammetry and Remote Sensing, 2014, 89: 59-66.

[62] Su W Z, Zhang Y, Yang Y B, Ye G B. Examining the impact of greenspace patterns on land surface temperature by coupling LiDAR data with a CFD Model. Sustainability, 2014, 6(10): 6799-6814.

[63] Middel A, Brazel A J, Gober P, Myint S W, Chang H, Duh J D. Land cover, climate, and the summer surface energy balance in Phoenix, AZ, and Portland, OR. International Journal of Climatology, 2012, 32(13): 2020-2032.

[64] Fahmy M, Sharples S, Yahiya M. LAI based trees selection for mid latitude urban developments: a microclimatic study in Cairo, Egypt. Building and Environment, 2010, 45(2): 345-357.

[65] Lin B S, Lin C T. Preliminary study of the influence of the spatial arrangement of urban parks on local temperature reduction. Urban Forestry \& Urban Greening, 2016, 20: 348-357.

[66] Huang H Y, Margulis S A, Chu C R, Tsai H C. Investigation of the impacts of vegetation distribution and evaporative cooling on synthetic urban daytime climate using a coupled LES—LSM model. Hydrological Processes, 2011, 25(10) : 1574-1586.

[67] Masoudi M, Tan P Y, Liew S C. Multi-city comparison of the relationships between spatial pattern and cooling effect of urban green spaces in four major Asian cities. Ecological Indicators, 2019, 98: 200-213.

[68] Wang L Y, Hou H, Weng J X. Ordinary least squares modelling of urban heat island intensity based on landscape composition and configuration: a comparative study among three megacities along the Yangtze River. Sustainable Cities and Society, 2020, 62: 102381.

[69] Naeem S, Cao C X, Qazi W A, Zamani M, Wei C, Acharya B K, Rehman A U. Studying the association between green space characteristics and land surface temperature for sustainable urban environments: an analysis of Beijing and Islamabad. ISPRS International Journal of Geo-Information, $2018,7(2): 38$.

[70] Estoque R C, Murayama Y, Myint S W. Effects of landscape composition and pattern on land surface temperature: an urban heat island study in the megacities of Southeast Asia. Science of the Total Environment, 2017, 577: 349-359.

[71] Fung C K W, Jim C Y. Microclimatic resilience of subtropical woodlands and urban-forest benefits. Urban Forestry \& Urban Greening, 2019, 42: 100-112.

[72] Saaroni H, Ben-Dor E, Bitan A, Potchter O. Spatial distribution and microscale characteristics of the urban heat island in Tel-Aviv, Israel. Landscape and Urban Planning, 2000, 48(1/2): 1-18.

[73] Arnfield A J. Two decades of urban climate research: a review of turbulence, exchanges of energy and water, and the urban heat island. International Journal of Climatology, 2003, 23(1): 1-26.

[74] Qaid A, Lamit H B, Ossen D R, Shahminan R N R. Urban heat island and thermal comfort conditions at micro-climate scale in a tropical planned city. Energy and Buildings, 2016, 133: 577-595.

[75] Ramamurthy P, Sangobanwo M. Inter-annual variability in urban heat island intensity over 10 major cities in the United States. Sustainable Cities and Society, 2016, 26: 65-75

[76] Georgi J N, Dimitriou D. The contribution of urban green spaces to the improvement of environment in cities: case study of Chania, Greece. Building and Environment, 2010, 45(6) : 1401-1414.

[77] Lin B S, Lin Y J. Cooling effect of shade trees with different characteristics in a subtropical Urban Park. HortScience, 2010, 45(1): 83-86.

[78] Mitchell V G, Cleugh H A, Grimmond C S B, Xu J. Linking urban water balance and energy balance models to analyse urban design options. 
Hydrological Processes, 2008, 22(16) : 2891-2900.

[79] Shashua-Bar L, Potchter O, Bitan A, Boltansky D, Yaakov Y. Microclimate modelling of street tree species effects within the varied urban morphology in the Mediterranean city of Tel Aviv, Israel. International Journal of Climatology, 2010, 30(1): 44-57.

[80] 王晓娟, 孔繁花, 尹海伟, 徐海龙, 李俊生, 蒲英霞. 高温天气植被蒸腾与遮荫降温效应的变化特征. 生态学报, 2018, 38 (12)： 4234- 4244.

[81] United Nations. World Urbanization Prospects: the 2014 Revision. New York: United Nations, 2014.

[82] Fouillet A, Rey G, Laurent F, Pavillon G, Bellec S, Guihenneuc-Jouyaux C, Clavel J, Jougla E, Hémon D. Excess mortality related to the August 2003 heat wave in France. International Archives of Occupational and Environmental Health, 2006, 80(1): 16-24.

[83] Tan J G, Zheng Y F, Tang X, Guo C Y, Li L P, Song G X, Zhen X R, Yuan D, Kalkstein A J, Li F R, Chen H. The urban heat island and its impact on heat waves and human health in Shanghai. International Journal of Biometeorology, 2010, 54( 1): 75-84.

[84] Bogdanović-Protić I S, Vukadinović A V, Radosavljević J M, Alizamir M, Mitković M P. Forecasting of outdoor thermal comfort index in urban open spaces: the Nis fortress case study. Thermal Science, 2016, 20( S5): S1531-S1539.

[85] Canan F, Golasi I, Ciancio V, Coppi M, Salata F. Outdoor thermal comfort conditions during summer in a cold semi-arid climate. A transversal field survey in Central Anatolia (Turkey). Building and Environment, 2019, 148: 212-224.

[86] Qian Y G, Zhou W Q, Li W F, Han L J. Understanding the dynamic of greenspace in the urbanized area of Beijing based on high resolution satellite images. Urban Forestry \& Urban Greening, 2015, 14(1) : 39-47.

[87] Lillesand T M, Kiefer R W, Chipman J W. Remote Sensing and Image Interpretation. New York: John Wiley \& Sons, 2004.

[88] Roberts D A, Dennison P E, Roth K L, Dudley K, Hulley G. Relationships between dominant plant species, fractional cover and Land Surface Temperature in a Mediterranean ecosystem. Remote Sensing of Environment, 2015, 167: 152-167.

[89] Jenerette G D, Harlan S L, Buyantuev A, Stefanov W L, Declet-Barreto J, Ruddell B L, Myint S W, Kaplan S, Li X X. Micro-scale urban surface temperatures are related to land-cover features and residential heat related health impacts in Phoenix, AZ USA. Landscape Ecology, 2016, 31(4) : $745-760$.

[90] Shashua-Bar L, Hoffman M E. Vegetation as a climatic component in the design of an urban street: an empirical model for predicting the cooling effect of urban green areas with trees. Energy and Buildings, 2000, 31(3): 221-235.

[91] Yu Z W, Xu S B, Zhang Y H, Jørgensen G, Vejre H. Strong contributions of local background climate to the cooling effect of urban green vegetation. Scientific Reports, 2018, 8: 6978

[92] Allegrini J, Carmeliet J. Coupled CFD and building energy simulations for studying the impacts of building height topology and buoyancy on local urban microclimates. Urban Climate, 2017, 21:278-305.

[93] Armson D, Rahman M A, Ennos A R. A comparison of the shading effectiveness of five different street tree species in Manchester, UK. Arboriculture \& Urban Forestry, 2013, 39(4) : 157-164. 